\title{
Spin Helicity in Chiral Lanthanide Chains
}

\author{
Ionut Mihalcea ${ }^{\ddagger}$, Mauro Perfetti ${ }^{\star, \dagger}$, Francesco Pineider $^{\dagger \star}$, Lorenzo Tesi ${ }^{\dagger}$, Valeriu Mereacre $^{\ddagger}$, \\ Fabrice Wilhelm ${ }^{\#}$, Andrei Rogalev ${ }^{\#}$, Christopher E. Anson ${ }^{\ddagger}$, Annie K. Powell ${ }^{\star} \$ \ddagger$, Roberta \\ Sessoli ${ }^{*, \dagger}$
}

$\ddagger$ Institute of Inorganic Chemistry, Karlsruhe Institute of Technology (KIT), Engesserstrasse 15, D-76131 Karlsruhe, Germany.

† Department of Chemistry “U. Schiff” Università di Firenze \& INSTM RU Firenze via della Lastruccia 3-13, 50019 Sesto Fiorentino, Italy.

' Department of Chemistry And Industrial Chemistry, University of Pisa, via Moruzzi 3, 56124 Pisa, Italy.

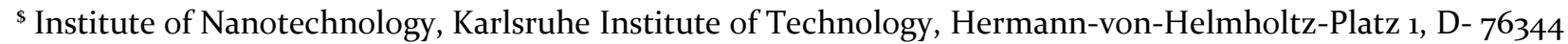

Eggenstein-Leopoldshafen, Germany.

"European Synchrotron Radiation Facility (ESRF), 38043, Grenoble, France.

\section{Supporting Information Placeholder}

\begin{abstract}
We report here the synthesis and determination of the helical spin structure of three Ln-based chiral chains of formula $\left[\operatorname{Ln}(\text { Hnic })(n i c)_{2}\left(\mathrm{NO}_{3}\right)\right]_{\mathrm{n}}$ (Hnic $=$ nicotinic acid, $\mathrm{Ln}=\mathrm{Tb}$, Dy, and $\mathrm{Er}$ ) by means of Cantilever Torque Magnetometry. The Tb $\mathrm{Tb}^{\mathrm{III}}$ and $\mathrm{Dy}{ }^{\mathrm{III}}$ ions exhibit strong noncollinear easy axis magnetic anisotropy, while easy plane anisotropy characterizes the $\mathrm{Er}^{\mathrm{III}}$ ions. Single crystals of the two enantiomers of the Dy derivative were also investigated using hard X-ray synchrotron radiation at the L-edge of the metal revealing optical activity, though with negligible involvement of the $4 f$ electrons of the Dy ion.
\end{abstract}

\section{INTRODUCTION}

The interplay between chirality and magnetism is one of the most unexplored topics in solid state physics. Even if the correlation between these two properties of matter was sensed since the $19^{\text {th }}$ century, ${ }^{1}$ a rational understating of the relation between them, known as magnetochiral effect (MChE), is still missing. The arising of MChE is possible in magnetoelectrical media and multiferroics, ${ }^{2}$ but also in magnetic systems when an external magnetic field is applied. Indeed, the observation of this effect, that is in general very challenging due to its intrinsic weakness, is possible only when the parity reversal and the time reversal symmetry are simultaneously broken. This is due to the fact that magnetic moments are generated by a circulation of electrons that is ruled by time-reversal symmetry while chirality is obviously parity odd. The literature reports on this effect are quite scarce in $2 \mathrm{D}$ and $3 \mathrm{D}$ architectures, 3 and, at the best of our knowledge, only one $1 \mathrm{D}$ system has been reported. 4 This last paper, published by some of us, testifies that chiral chains based on transition metal ions (TM) can be good candidates to observe this effect using synchrotron hard X-rays. What emerged from the study of these metal-nitronyl nitroxide chains ${ }^{4}$ is that the main ingredients to have a strong MChE are high mixing between orbitals of different parity, allowed in non-centrosymmetric structures, and a strong magnetic anisotropy. In fact, while the $\mathrm{Co}^{\mathrm{II}}$ derivative, provided a giant magneto-chiral dichroism, the signal was practically absent in the isotropic $\mathrm{Mn}^{\mathrm{II}}$ derivative. What remained still unclear is if the spin helicity played a significant role. Indeed, only the $\mathrm{Co}^{\mathrm{II}}$ derivative exhibited a nonzero angle between the chain axis and the easy anisotropy axis of the magnetization of the metal centers.

Shifting from TM to lanthanides ( $\mathrm{Ln})$ an increase of the magnetic anisotropy and the orbital contribution to the magnetization are expected. Moreover, $L n^{\mathrm{III}}$ ions exhibit a magnetic anisotropy that can vary significantly in nature and orientation also along an isostructural series. This variation is often explained by simple electrostatic considerations about the electronic density generated by the partially filled $4 f$ orbitals around the metal center. 5

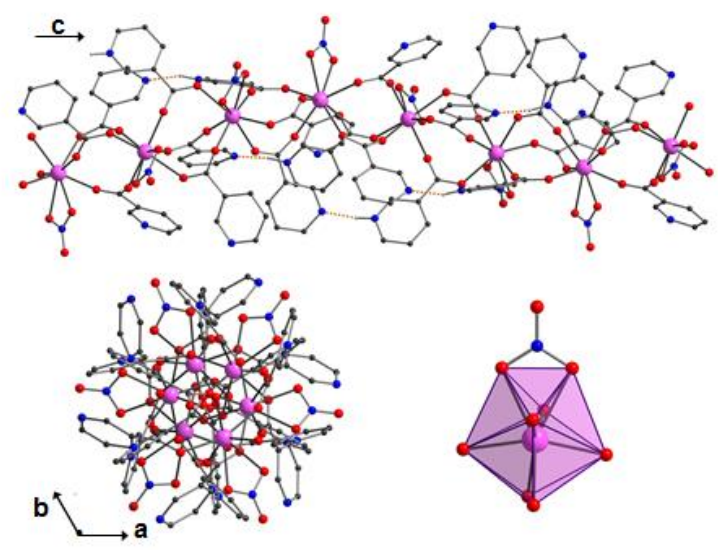

Figure 1: The structure of the helical chain in Er viewed from the side (above) and down the 65 axis (below left), and 
the coordination polyhedron of $\operatorname{Er}(1)$ showing its idealised dodecahedral C2v symmetry (below right). Er pink, $\mathrm{O}$ red, $\mathrm{N}$ blue, $\mathrm{C}$ gray; organic $\mathrm{H}$ omitted for clarity, $\mathrm{H}$-bonds dashed orange lines.

Some of us recently reported the synthesis, structural and magnetic characterization of a series of chiral chains of formula $\left[\mathrm{Ln}(\mathrm{Hnic})(\mathrm{nic})_{2}\left(\mathrm{NO}_{3}\right)\right]_{\mathrm{n}}$ where Hnic is nicotinic acid and $\mathrm{Ln}=$ the $\mathrm{Eu}, \mathrm{Gd}$, and $\mathrm{Tb} .{ }^{6}$ Despite the absence of chiral ligands, spontaneous resolution of the two helicities occurs through crystallization in the enantiomeric $\mathrm{P}_{1}$ and $\mathrm{P}_{5}$ space groups; Flack $\chi_{\mathrm{F}}$ parameters of zero from the structure refinements demonstrate that any one crystal is enantiomerically pure. Magnetic studies revealed a weak ferromagnetic Gd-Gd interaction. With the purpose to investigate the correlation between electronic structure and presence/absence of MChE, in this paper we analyze the spin structure of the Tb, Dy and Er members of this series. In Figure 1 we reported the structure of the Er derivative. While this work was in progress, Liu et al. published the structure and magnetic data of the isotypic Dy analogue, which showed slow relaxation of its magnetization.7

Among the Ln, we focused on the highly anisotropic ions $\mathrm{Tb}(\mathrm{III}), \operatorname{Dy}(\mathrm{III})$ and $\operatorname{Er}(\mathrm{III})(J=6, J=15 / 2$ and $J=15 / 2$ ground states, respectively). In order to disentangle the single ion contributions to the magnetic anisotropy and to gain insight in the spin helicity we exploited the high sensitivity of Cantilever Torque Magnetometry (CTM). ${ }^{8}$ Moreover, we investigated the natural circular dichroism irradiating single crystals with hard X-ray synchrotron radiation at the Ln metal L- edge.

\section{EXPERIMENTAL SECTION}

Crystals of $\left[\mathrm{Ln}(\mathrm{Hnic})(\mathrm{nic})_{2}\left(\mathrm{NO}_{3}\right)\right]_{\mathrm{n}}$, hereafter labelled with the respective lanthanide atomic symbol in bold ( $\mathbf{T b}, \mathbf{D y}, \mathbf{E r})$ as well as the derivative where Dy is diluted in the diamagnetic Y analogue (DyY) were prepared according to the reported method. For DyY a 1:5 molar ratio of Dy $\left(\mathrm{NO}_{3}\right)_{3} \cdot 6 \mathrm{H}_{2} \mathrm{O}$ and $\mathrm{Y}\left(\mathrm{NO}_{3}\right)_{3} \cdot 6 \mathrm{H}_{2} \mathrm{O}$ was used.

The crystal structures of Er and DyY were determined using $\mathrm{Cu}-\mathrm{K} \alpha$ (Oxford Diffraction SuperNova E) and Mo-Ka (Bruker SMART Apex) radiation, respectively, and found to be isotypic with the Eu, Gd, Tb and Dy analogues. The metal ion in DyY was refined as a mixture of Y and Dy. With rigid bond restraints to the thermal parameters of the metal and surrounding oxygens, the s.o.f. for Dy refined to $11.9(3) \%$ (in good agreement with the value of $15 \%$ from the magnetic data) and the composition was fixed at $88 \% \mathrm{Y}$ and $12 \%$ Dy in final refinements. As before, both the presence of merohedral twinning by $180^{\circ}$ rotation about $\mathbf{a}+\mathbf{b}$ (which does not change the chirality) and of twinning by inversion were checked; For Er and DyY, the merohedral twinning amounted to $0.248(1)$ and $0.0205(4)$, and the Flack $\chi_{F}$ parameters (both from 100\% Friedel coverage) were -o.o16(6) and -0.012(3), respectively, showing that the crystals used were, once again, enantiomerically pure.

Er: $\mathrm{C}_{18} \mathrm{H}_{13} \mathrm{ErN}_{4} \mathrm{O}_{9}, 596.58 \mathrm{~g} \mathrm{~mol}^{-1}$, hexagonal, $\mathrm{P}_{5}, a=$ 11.1520(2), $c=26.4830(4) \AA, Z=6, V=2852.35(13) \AA 33, \mathrm{~T}=$ $100(2) \mathrm{K}, \rho_{\text {calc }}=2.084 \mathrm{~g} \mathrm{~cm}^{-3}, F($ ooo $)=1734, \mu(\mathrm{Cu}-\mathrm{K \alpha})=8.763$ $\mathrm{mm}^{-1} ; 15510$ data, 3717 unique $\left(R_{\mathrm{int}}=0.0384\right), 294$ parameters, final $w R_{2}=0.0435, S=1.062$ (all data), $R_{1}$ (3699 data with I >
$2 \sigma(\mathrm{I}))=0.0179$, Flack $\chi_{\mathrm{F}}=-0.016(6)$, max. Peak/hole in final diff. map $=+0.32 /-0.33$ e $\AA^{-3}$.

DyY: $\mathrm{C}_{18} \mathrm{H}_{13} \mathrm{Dy}_{0.12} \mathrm{~N}_{4} \mathrm{O}_{9} \mathrm{Y}_{0.88}, 527.06 \mathrm{~g} \mathrm{~mol}^{-1}$, hexagonal, $\mathrm{P}_{5}$, $a=11.1664(3), c=26.5948(18) \AA, Z=6, V=2871.8(2) \AA 33, \mathrm{~T}=$

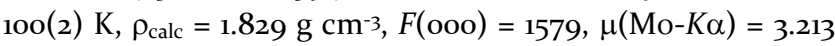
$\mathrm{mm}^{-1} ; 24095$ data, 4421 unique $\left(R_{\text {int }}=0.0299\right), 294$ parameters, final $w R_{2}=0.0486, S=1.039$ (all data), $R_{1}(4315$ data with $\mathrm{I}>2 \sigma(\mathrm{I}))=0.0207$, Flack $\chi=-0.012(3)$, max. Peak/hole in final diff. map $=+0.64 /-0.33$ e $\AA$-3.

Crystallographic data for the structures of Er and DyY have been deposited with the Cambridge Crystallographic Data Center as supplementary publications CCDC 1473784 and 1473785. Copies of the data can be obtained from: https://summary.ccdc.cam.ac.uk/structure-summary-form.

The cantilever torque measurements were performed using a home made two-legs $\mathrm{CuBe}$ cantilever separated by o.1 $\mathrm{mm}$ from a gold plate. The cantilever was inserted in an Oxford Instruments MAGLAB20oo platform with automated rotation of the cantilever chip in a vertical magnet. The capacitance of the cantilever was detected with a AndeenHegerling 2500A Ultra Precision Capacitance Bridge. A more detailed sketch of the experimental setup is reported in Figure $\mathrm{S}$.

The static and dynamic magnetic properties were measured on crystalline powders in form of pressed pellets in the Quantum Design MPMS SQUID magnetometer.

X-ray Absorption Near-Edge Fine Structure (XANES) and X-ray Natural Circular Dichroism (XNCD) experiments at the L3 edge of Dy were carried out at the ID12 beamline of the European Synchcrotron Radiation Facility (Grenoble, F), dedicated to polarization-dependent X-ray spectroscopy in the 2-15 keV energy range. 9 A high flux of circularly polarized photons was provided by an APPLE-II helical undulator. Xrays were monochromatized by a $\mathrm{Si}<111>$ double crystal monochromator. Samples were mounted on an aluminum sample holder and oriented so that the crystal's c axis and the photons' wave vector formed an angle of approximately $18^{\circ}$. All spectra were recorded in total X-ray fluorescence mode in backscattering geometry. Each set of XNCD spectra was acquired by alternating photon helicity 18 times, for a total of 36 XANES spectra.

\section{RESULTS AND DISCUSSION}

\section{Torque magnetometry}

The determination of the principal directions of anisotropy in crystals with noncollinear anisotropy tensors can be achieved using CTM, as already reported in the literature for mononuclear lanthanide compounds ${ }^{10}$ and TM clusters. ${ }^{11}$ The technique requires indexed single crystals fixed on the upper plate of a capacitor that can be rotated in a static magnetic field (B). When the temperature (T) is lowered enough, it is possible to detect the torque $(\tau)$ acting on the sample as a change in capacitance. In the low field limit $\left(g \mu_{B} B<<k_{B} T\right)$ the torque along a fixed direction (e.g. the $Y$ axis in the laboratory $X Y Z$ frame) is connected to the susceptibility tensor $(\chi)$ by the following relation:

$$
\tau_{Y}=M_{Z} B_{X}-M_{X} B_{Z}=B^{2}\left(\chi_{z Z}-\chi_{X X}\right) \sin \varphi \cos \varphi
$$


Where $\varphi$ is the angle between the projection of the $z$ molecular axis in the $X Z$ plane and $\mathbf{B}$. Note that this angle is in general not coincident with the angle of rotation of the cantilever, $\theta$. An additional term dependent on $\chi x \mathrm{xz}$ was omitted because it is always possible to rotate the reference frame and set it to zero. When the field is increased, the response is no more tensorial so that torque curves deform such that the angular dependence becomes very different if the easy axis is almost parallel or perpendicular to the field allowing for the deconvolution of the contribution of noncollinear centers as their symmetry-related contributions do not cancel out completely.

This approach has been used to extract information on the single ion anisotropy in the chiral coordination polymers investigated here. Since the $P 6_{1} / P 6_{5}$ enantiomeric space groups possess a $\mathrm{C}_{6}$ axis parallel to the crystallographic $c$ axis, assuming uniaxial anisotropy of the individual metal ions, this exotic structure imposes that the orientation of the anisotropy tensors of the six crystallographically equivalent ions can be determined using two relevant Euler angles, e.g. the angle between the $z$ molecular axis and the $c$ axis $(\xi)$, that is equal for all the spins, and the angle between the $a$ axis and the projection of the $z$ molecular axis on the $a b$ plane $(\rho)$. When the principal directions of the first anisotropy tensor are fixed, the others can be easily determined by adding a factor $(60 \cdot i)^{\circ}$ (where $i=1$ to 5 ) to $\rho$. It is important to notice here that the helicity of the spin chain is only dependent on the value of the angle $\xi$, in particular it is present for any value of this angle different from $0^{\circ}$.

All data were recorded varying the angle between $\boldsymbol{B}$ and the upper plate of the cantilever $(\theta)$ at fixed temperature (see Figure $\mathrm{S}_{1}$ ). For each derivative two rotations were performed. During the first one (Rot1) the rotation axis was the $c$ axis, while in the second one (Rot2) the rotation axis lay in the $a b^{\prime}$ plane (see Figure $\mathrm{S}_{2}$ for a detailed position of all the crystals). This set of rotations is particularly useful to determine the Euler angles because Rotı is particularly sensitive to $\rho$ while Rot 2 is extremely dependent on $\xi$ (see Figure $S_{3}$ for sample calculations evidencing the dependence of the crystal torque signal on the $\xi$ and $\rho$ angles).

In Figure 2 we report the torque curves for $\mathbf{T b}, \mathbf{D y}$ and $\mathbf{E r}$ in the relevant $\left(0-180^{\circ}\right)$ angular range. Other curves recorded at different temperatures and fields are reported in Figure $\mathrm{S}_{4}$, $\mathrm{S}_{5}$ and S6. We also investigated a crystal of the DyY sample. The obtained torque curves, reported in Figure $S_{7}$, are very similar to those of the pure Dy. This is not surprising since the very weak ferromagnetic interaction (vide infra) is not affecting the magnetic behavior of the system in the relatively strong magnetic field employed in the torque measurements.

Looking at Figure 2, it is evident that all samples exhibit the six-fold symmetry imposed by the structure. Rotı has in fact six zero-torque points that correspond to the three independent projections on the $a b$ plane of the six anisotropy tensors (we recall here that a $180^{\circ}$ rotation does not affect the anisotropy) arising from the $\mathrm{Ln}$ ions, and all the maxima have similar intensity, as expected when the rotation axis coincides with $c$. Rot2 has more complicated features that differ for the three compounds, but the signal always vanishes near $0^{\circ}$ and $90^{\circ}$, corresponding to $c / / \mathbf{B}$ and $c \perp \mathbf{B}$, respectively.

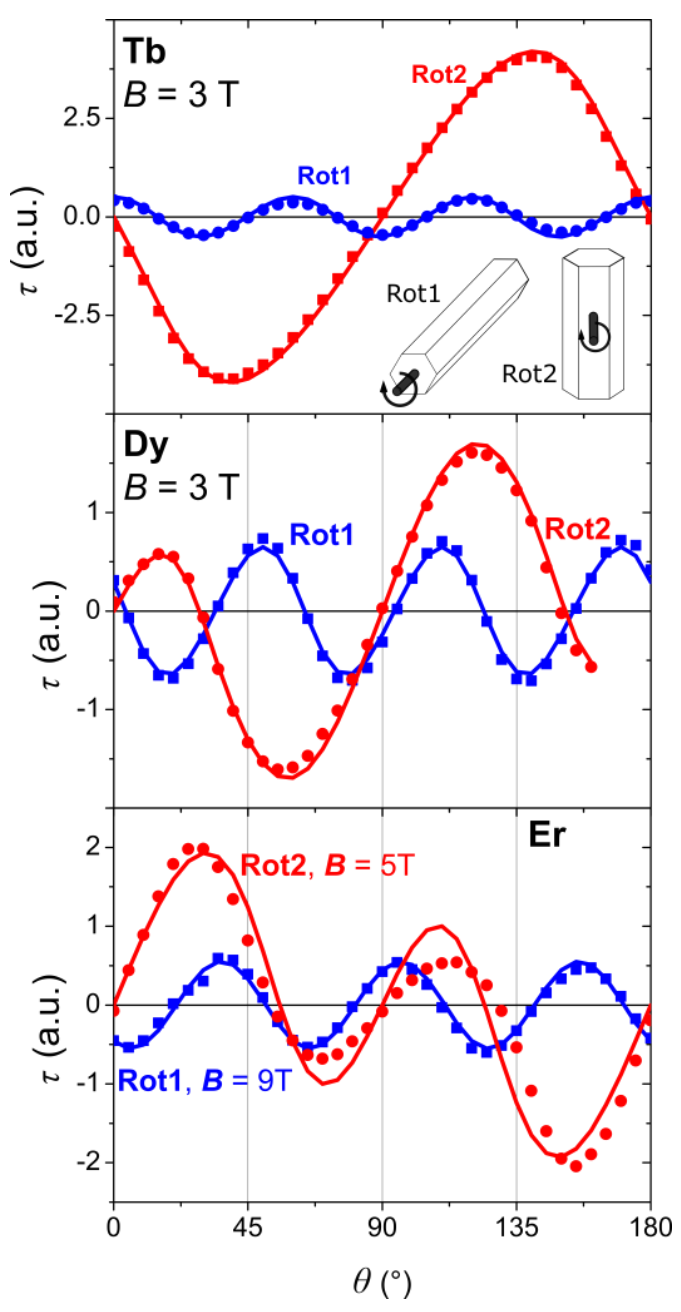

Figure 2. Experimental (dots or squares) and fitted (straight lines) curves obtained for Tb (up), Dy (middle) and for $\mathbf{E r}$ (down).

To extract the single ion magnetic anisotropy modellization including both the Zeeman and the anisotropy term is necessary. The latter at the simplest level can be described by a crystal field (CF) potential acting on the metal center using the following Hamiltonian:

$$
\mathcal{H}=g_{L} \mu_{B} \boldsymbol{B} \cdot \hat{\boldsymbol{J}}+\sum_{k=2,4,6} \sum_{q=-k}^{k} b_{k}^{q} \widehat{\boldsymbol{o}}_{k}^{q}
$$

Where $g_{L}$ is the Landè factor of the Ln ion, $\mu_{B}$ is the Bohr magneton, $\boldsymbol{J}$ is the total angular momentum operator, $b^{q}$ are the coefficients of the $\boldsymbol{O}^{q}{ }_{k}$ Stevens operators ${ }^{12}$ that act on the $J, \mathrm{~m}_{\rho}>$ space, where $m_{J}$ is the projection of $J$ in the $z$ direction.

The experimental points were reproduced by adjusting the $b^{\mathrm{o}}{ }_{2}$ parameter and letting the two Euler angles vary freely (Figure 2). Also a scale factor was included in the fit to take into account the inaccuracy in the mass determination of such small crystals (accordingly to that the torque is given in a.u.). The inclusion of a single CF operator gave satisfactory fits for Dy and $\mathbf{E r}$, while for $\mathbf{T b}$ the inclusion of $\mathrm{a} \mathrm{O}_{{ }_{2}}$ operator was necessary. It is clear that using only few $\mathrm{CF}$ parameters is not sufficient to fully describe the magnetic 
anisotropy of these complexes, that are characterized by a low symmetry coordination environment. Moreover, the low temperature regime of all measurements does not allow us to extract information on the energy of excited Stark sublevels, so the values of the $\mathrm{CF}$ parameters given here are only accurate in providing information on the low temperature magnetic anisotropy. The simulation of the experimental torque data provided however the sign of the $b^{\circ}{ }_{2}$ parameter thus suggesting the nature, easy axis or easy plane, of the magnetic anisotropy of the metal ions. The best fit was achieved fixing a negative $b^{\circ}{ }_{2}$ for Tb $\left(b^{\circ}{ }_{2}=-1.54 \mathrm{~cm}^{-1},\right)$ and Dy $\left(b^{\circ}{ }_{2}=-1.91 \mathrm{~cm}^{-1}\right)$, therefore both characterized by single ion easy axis anisotropy, while an easy plane anisotropy was detected for $\mathbf{E r}\left(b^{\circ}{ }_{2}=+2.33 \mathrm{~cm}^{-1}\right)$. In the case of $\mathbf{T b}$, the inclusion of a transverse term $\mathrm{O}_{2}{ }_{2} \quad\left(b^{2}{ }_{2}=3.8 \mathrm{~cm}^{-1}\right)$ in the Hamiltonian, makes in principle the use of a third Euler angle $\psi=23(1)^{\circ}$ mandatory. A similar sign of magnetic anisotropy for $\mathbf{T b}$ and Dy, and an opposite one for $\mathbf{E r}$, is not unexpected since the electronic density of the $4 f$ shell can be approximated as an oblate spheroid in the case of $\mathrm{Tb}^{\mathrm{III}}$ and Dy III and prolate spheroid for $\mathrm{Er}^{\mathrm{III}} .5$ The obtained energy splitting of the ground $J$ multiplet is reported for all derivatives in Figure S8.

The best fit values of Euler angles were $\rho=31(1)^{\circ}, \quad \xi=76(1)^{\circ}$ for Tb, $\rho=41(1)^{\circ}, \xi=61(1)^{\circ}$ for Dy and $\rho=21(1)^{\circ}, \xi=56(1)^{\circ}$ for Er. The values of $\xi$ deserve some comments. All the values are larger than $54.7^{\circ}$ (magic angle), indicating that the $c$ crystallographic axis is closer to the hard direction in $\mathbf{T b}$ and Dy and to the easy direction in Er. The largest difference between the signals of the two rotations is observed for $\mathbf{T b}$, that has in fact the largest value of $\xi$.

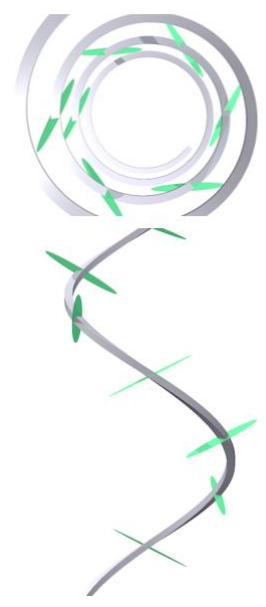

Tb

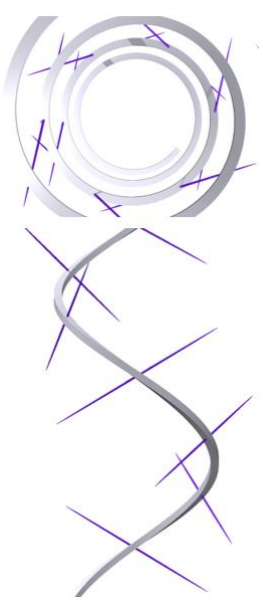

Dy

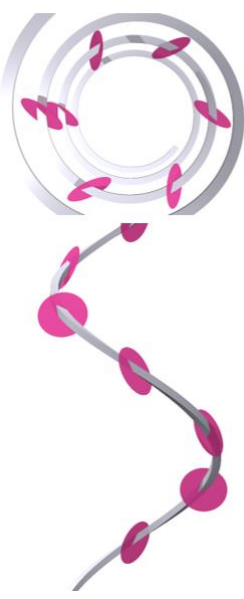

Er
Figure 3. Schematic representation of the spin helicity obtained by plotting the susceptibility tensors calculated from the CTM experiments at T $=5 \mathrm{~K}$ and $\boldsymbol{H}=\mathbf{1}$ Oe (up: view along $c$, down: view along the $a b$ plane). The susceptibility of the hard directions ( $x$ and $y$ ) of Dy has been multiplied by a factor of 5 to give a more solid aspect to the ellipsoids.

Interestingly, for the three helices the observed spin arrangement reflects in an overall magnetic anisotropy of the crystals which is reversed compared to that of the single ions: easy $a b$ plane of magnetization for $\mathbf{T b}$ and Dy and easy $c$ axis for $\mathbf{E r}$, as shown by the single crystal $M v s H$ curves reported in Figure S9. Moreover, the overall crystal anisotropy of Dy and $\mathbf{E r}$ is very weak because the angle $\xi$, is close to the magic angle.

In Figure 3 we provide a graphic representation of the spin helicity of the three investigated chains where the anisotropy susceptibility tensors generated by the six-fold screw axis are depicted. The CF parameters determined by CTM were employed to calculate the low field susceptibility tensors at $\mathrm{T}=5 \mathrm{~K}$. To make the strongly axial tensor of Dy visible, the hard directions of the tensor reported in Figure 3 where multiplied by a factor 5 .

Though CTM is very powerful in disentangling the single ions contributions, additional information is necessary to associate the extracted Euler's angles to a specific site, and a six-fold ambiguity remains on the $\rho$ value. A simple electrostatic modelling of the Dy(III) ion coordination environment with Magellan software ${ }^{13}$ provides a theoretical $\xi$ value close to $81^{\circ}$, significantly different from the experimental one $\left(61^{\circ}\right)$. More accurate approaches, able to take into account also the covalent character of the interaction, are necessary to reproduce the correct anisotropy orientation, but beyond the scope of this paper.

\section{Static and Dynamic magnetic properties}

Having successfully extracted the single ion anisotropy out of the almost isotropic crystals, it is interesting to investigate if the detected large single ion magnetic anisotropy correlates with the dynamics properties of the three helices. In agreement with the Kramers nature of the ion and its easy axis anisotropy stabilizing the $m_{J}= \pm 15 / 2$ doublet, Dy exhibits slow relaxation of the magnetization in zero static field, which further slows down in an applied field, as already reported by Liu et al..7 We investigated here the ac susceptibility of the other two lanthanide helices in zero and static applied field. Interestingly, neither $\mathbf{T b}$, which shows a significant rhombic anisotropy with the ground singlet separated by $2.4 \mathrm{~cm}^{-1}$ to the first excited state (see Figure S8 for energy and compositions of the lower states), nor $\mathbf{E r}$, with easy plane single ion anisotropy (ground $m_{J}= \pm \mathbf{1} / 2$ ), present SMM behavior.

Static magnetic data have evidenced an upturn at low temperature of the $\chi \mathrm{T}$ product for the $\mathrm{Gd}^{6}$ and $\mathrm{Dy}^{7}$ derivative indicative of weak ferromagnetic interactions. In contrast $\mathrm{Tb}^{6}$ only showed a monotonous decrease of $\chi \mathrm{T}$ on decreasing temperature. An almost monotonous decrease of $\chi \mathrm{T}$ is also observed for Er (see Figure S1o) suggesting that interactions between paramagnetic centers are practically negligible in this derivative.

Magnetically coupled centers with strong easy axis magnetic anisotropy are characterized by slow dynamics of the magnetization, known as Single Chain Magnet (SCM) behavior. In general, the effective barrier for the reversal of magnetization are higher in SCM as the exchange interaction contributes significantly to the energy cost of the relaxation process. Most reports of SCM, including the archetypal CoPhOMe, are based on transition metal ions There are a few reports of slow magnetization dynamics in onedimensional structures comprising Dy ions, ${ }^{14}$ but an assessment of the role of the weak exchange interaction in the slow dynamics of the magnetization is not 
straightforward. For this purpose, we investigated the diluted DyY where the diluition percentage (15\%) assures that most Dy ions are connected to two diamagnetic neighbors. Figure 4 reports the temperature dependence in $1 \mathrm{kOe}$ field of the $\chi \mathrm{T}$ per mole of Dy(III) ion for pure and diluted samples. The upturn is not observable for the DyY sample, as expected.

To quantify the interaction active in the pure chain the low temperature $\chi \mathrm{T}$ data were reported in a $\log$ vs $1 / \mathrm{T}$ plot. Linearity is observed with a slope of o.33(1) K (see Figure S11). In the Ising limit the slope of the semi-logarithmic plot corresponds to the interaction energy, i.e. the difference in energy for parallel and antiparallel alignment of neighboring spins. Interestingly, this value is not too dissimilar to that found for the Gd derivative, where $E_{\text {exch }}=2 j S^{2}=0.46 \mathrm{~K}$ for a coupling constant $j=0.013 \mathrm{~cm}^{-1}$ with the interaction Hamiltonian written as $\mathscr{H}_{\text {int }}=j S_{a} \cdot S_{b} \cdot{ }^{6}$ As expected for pure $4 f$ systems, the estimated interactions are very weak and in the present case comparable to dipolar contributions. Suppression of ferromagnetic interactions in $\mathrm{Tb}$ and $\mathrm{Er}$ derivatives, in contrast to Gd and Dy ones, could actually be originated by a different contribution of dipolar interactions, given the different orientation of the magnetic moments in the chains.

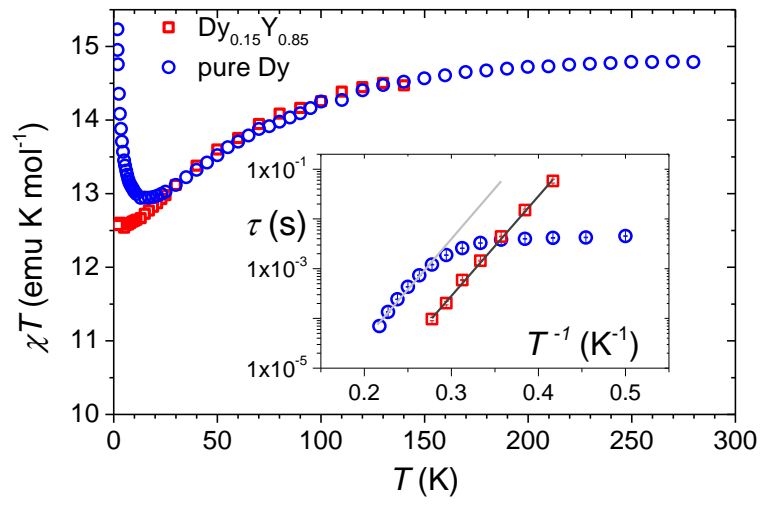

Figure 4. Temperature dependence of the $\chi \mathrm{T}$ product for the pure and diluted Dy helices measured applying $\boldsymbol{H}=1000$ Oe. In the inset the relaxation times for the two samples in the same static field. Gray and black lines correspond to the best fit with Arrhenius law of the data in the linear regime (see text).

The dynamic behavior of the pure and diluted Dy helices was also investigated by ac susceptometry. A static field of 1000 Oe was employed to slow down the magnetization. Maxima in the imaginary component the susceptibility vs. frequency were observed below $4.5 \mathrm{~K}$ for Dy and $3.8 \mathrm{~K}$ for YDy (Figure S12, S13 and S14). Data analyzed with the Debye model provided the temperature dependence of the relaxation times reported as an Arrhenius plot in the inset of Figure 4. Both samples exhibit a linear high temperature region, with levelling of the relaxation time at low temperature. This effect is far more pronounced in the pure sample, indicating that spin-spin interactions are promoting fast relaxation. This observation is in contrast with what expected for SCM, suggesting that the magnetization dynamics is still dominated by the single ion anisotropy.
Fitting of the linear regime provided the parameters of the Arrhenius law, $\tau=\tau_{\mathrm{o}} \exp \left(\Delta \mathrm{E} / \mathrm{k}_{\mathrm{B}} \mathrm{T}\right): \Delta \mathrm{E}=47(2) \mathrm{K}, \tau_{\mathrm{o}}=3(1) 10^{-9} \mathrm{~s}$ for Dy and $\Delta \mathrm{E}=46.2(8) \mathrm{K}, \tau_{\mathrm{o}}=2.7(7) 10^{-10} \mathrm{~s}$ for $\mathrm{YDy}$, respectively. The difference in the effective barrier is within the experimental error which is, however, comparable to the expected contribution deriving from the intrachain interaction (note also the similar width of the relaxation times distribution reported in Figure $\mathrm{S}_{15}$ ). Significant is, however, the difference of almost an order of magnitude in the pre-exponential factor, with the longer $\tau_{\mathrm{o}}$ observed for the pure sample. The decrease of $\tau_{\mathrm{o}}$ with diamagnetic doping was already observed in $\mathrm{SCM}^{15}$ and attributed to the increase of the number of defective where (i.e. those with a diamagnetic neighbor) where the domain walls nucleate because of the reduced exchange energy cost. However, this picture cannot be reconciled to our finding, because of the almost negligible contribution to the spin dynamics arising from by magnetic interaction along the chain. An alternative explanation could be that by magnetic dilution more phonons become available to promote magnetic relaxation. Acceleration of the relaxation is in fact observed in diluted samples when spin-phonon bottleneck effects are present, but further experiments at different dilutions of the Dy magnetic moments are necessary to elucidate this point.

\section{X-ray Natural Circular Dichroism}

Our investigation gives evidence for a spin helicity in the Dy coordination polymer very similar to that detected in the Co(II) nitronyl-nitroxide chain exhibiting strong X-ray natural and magnetic circular dichroisms as well as giant $\mathrm{X}$ ray magneto-chiral dichroism at the K-edge of Co. With the optical activity of the Dy crystals already assessed (by Liu et al?) we decided to investigate the lanthanide contribution to it exploiting the element sensitivity of X-ray spectroscopy. The X-ray absorption (XANES) and natural circular dichroism (XNCD) measurements have been attempted on Dy crystals at the $\mathrm{L}_{3}$ edge of Dy, i.e. optical transitions from the $2 \mathrm{p} 3 / 2$ core orbitals to unoccupied states, were therefore studied.

This is not the edge usually employed to access the magnetic properties of the $4 \mathrm{f}$ states of lanthanides since at the $\mathrm{L}_{3}$ absorption edge of a rare-earth atom the dominant electric dipolar (E1) transitions are towards unoccupied $5 \mathrm{~d}$ orbitals and electric quadrupolar (E2) transitions to partially filled 4 f orbitals and to empty $6 \mathrm{p}$ orbitals are much weaker. It should be recalled that the term of matter-radiation interaction leading to optical activity, i.e. natural circular dichroism, in the X-ray range is an interference dipolequadrupole term (E1E2) which averages to zero in randomly oriented samples and could be only detected on systems featuring an orientational order, e.g single crystals. 


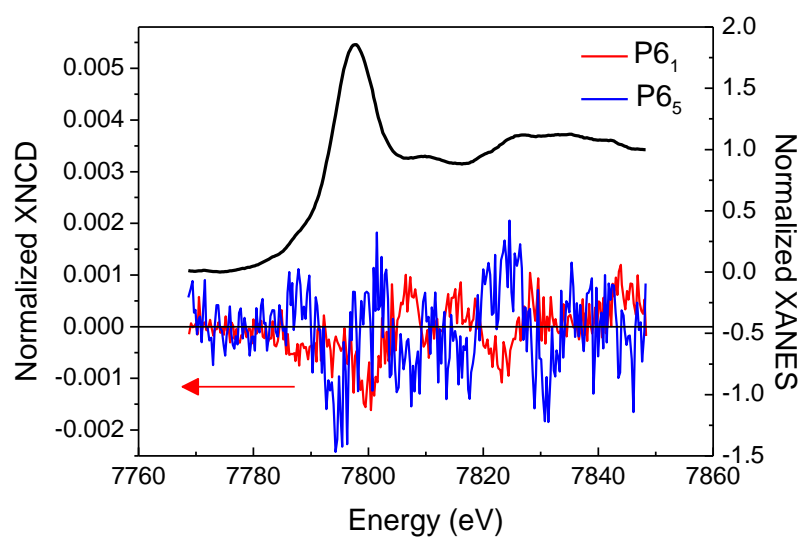

Figure 5. Near edge X-ray absorption spectrum (XANES) at the $\mathrm{L}_{3}$ edge of Dy measured at room temperature in zero field (black line) obtained as $1 / 2\left(\sigma^{\mathrm{r}}+\sigma^{\mathrm{l}}\right)$ and X-ray natural circular dichroism spectra given by $\left(\sigma^{\mathrm{r}}-\sigma^{\mathrm{l}}\right)$ for two enantiomeric crystals. $\sigma^{\mathrm{r}}$ and $\sigma^{\mathrm{l}}$ denotes $\mathrm{x}$-ray absorption cross section for right- and left- circularly polarized x-rays, respectively.

Two relatively large (ca. $2.5 \times 0.2 \times 0.2 \mathrm{~mm}$ ), enantiopure single crystals of opposite chirality (P61, Flack $\chi=-0,016(8)$; P65, Flack $\chi=-0.017(6))$ were selected for X-ray absorption experiments. The Dy L3-edge XANES and XNCD spectra were measured at room temperature and shown in Figure 5. The isotropic contribution to the X-ray Absorption (XANES) is dominated by the characteristic intense peak due to transitions into $5 \mathrm{~d}$ empty orbitals, the so-called "white line" with maximum at $7795 \mathrm{eV}$. Quadrupolar transitions into unoccupied $4 \mathrm{f}$ orbitals occur at about 5-10 eV lower in energies due to a strong interaction of the $2 \mathrm{p} 3 / 2$ hole and $4 \mathrm{f}$ electrons, which could be hardly seen in isotropic XANES spectra. On the other hand these transitions are nicely observable in the corresponding X-ray magnetic circular dichroism spectra. ${ }^{16}$ A rather weak XNCD signal is observed at the Dy L3-edge, with opposite sign for the P61 and P65 enantiomeric crystals. The magnitude of the normalized $\mathrm{XNCD}$ signal is in the low $10^{-3}$ range, similarly to previous reports on XNCD at the $\mathrm{L}$ edge of lanthanide chiral complexes. ${ }^{17}$ The relatively weak dichroism can reasonably be ascribed to the fact that $f$ orbitals are strongly localized on the lanthanide ion, thus are scarcely influenced by a supramolecular chiral arrangement such as that of the nicotinate helix studied in this work. This is also confirmed by the very weak XNCD intensities at photon energies where electronic transitions to states with a strong $4 \mathrm{f}$ character is expected (ca. $7787 \mathrm{eV}$ ). On the other hand, quadrupolar transitions at the $\mathrm{L}_{3}$ edge to extended $6 \mathrm{p}$ states of rare-earth are also allowed by the selection rules, thus giving rise to a finite XNCD signal in wide energy range up to $30 \mathrm{eV}$ above the edge. These extended states are indeed strongly affected by the chiral arrangement of ligands. In this respect $x$-ray optical activity at the L3-edge of rare-earth in Dy helices strongly differs from previously investigated $\mathrm{Co}^{\mathrm{II}}$ and $\mathrm{Mn}^{\mathrm{II}}$ ones which revealed a large natural circular dichroism signal due to interference of $1 s \rightarrow 3$ d quadrupolar and $1 s \rightarrow 4$ p dipolar transitions. These findings suggest that, despite the fact that strong spin noncollinearity of lanthanide-based helices makes them strong candidates for the observation of magnetochiral effects, use of hard X-rays does not appear to be straightforward for such investigations.

\section{CONCLUSIONS}

In conclusion, we have exploited the power of cantilever torque magnetometry to investigate the spin helicity of a series of isomorphous coordination polymers, extracting precious information on the single ion magnetic anisotropy. All derivatives exhibit spin noncollinearity, thus a marked spin helicity, though only the Dy derivative has the correct anisotropy to promote slow relaxation of the magnetization. The synchrotron measurements also suggested a weak admixing of the $4 f$ orbitals with orbitals of opposite parity, thus hampering the detection of a significant MChE with this technique. This however does not preclude the observation of MChD in the NIR-visible region, though the necessity to use single crystals poses additional experimental difficulties that we are currently trying to solve.

\section{ASSOCIATED CONTENT}

\section{Supporting Information}

Details about the synthesis and about the used experimental techniques. Simulations of torque curves at different conditions and other fits. Energy diagrams, AC and DC magnetometry.

\section{AUTHOR INFORMATION}

\section{Corresponding Authors}

* email: mauro.perfetti@ipc.uni-stuttgart.de

* email: roberta.sessoli@unifi.it

* email: annie.powell@kit.edu

\section{Present Addresses}

$\int$ Institut für Physikalische Chemie, Pfaffenwaldring 55, 70569, Stuttgart, Deutschland.

\section{Funding Sources}

(Word Style "Section_Content"). Any funds used to support the research of the manuscript should be placed here (per journal

style).

\section{Notes}

The authors declare no competing financial interests.

\section{ACKNOWLEDGMENT}

We thank Lena Friedrich for assistance with the synthesis. IM, VM, CEA and AKP thank the DFG-funded transregional collaborative research center SFB/TRR 88 "3MET" for funding. The European Synchrotron Radiation Facility is acknowledged for providing beamtime (experiment $\mathrm{HC}$ 1810). 


\section{ABBREVIATIONS}

(Word Style "Section_Content"). CCR2, CC chemokine receptor 2; CCL2, CC chemokine ligand 2; CCR5, CC chemokine receptor 5 ; TLC, thin layer chromatography.

\section{REFERENCES}

1. Pasteur, L., La dissymétrie moléculaire. Rev. Sci. 1884, 7 .

2. (a) Cheong, S.-W.; Mostovoy, M., Multiferroics: a magnetic twist for ferroelectricity. Nat Mater 2007, 6 (1), 1320; (b) Khomskii, D., Trend: Classifying multiferroics: Mechanisms and effects. Physics 2009, 2, 20.

3. (a) Train, C.; Gheorghe, R.; Krstic, V.; Chamoreau, L.-M.; Ovanesyan, N. S.; Rikken, G. L. J. A.; Gruselle, M.; Verdaguer, M., Strong magneto-chiral dichroism in enantiopure chiral ferromagnets. Nat. Mater. 2oo8, 7 (9), 729-734; (b) Kubota, M.; Arima, T.; Kaneko, Y.; He, J.; Yu, X.; Tokura, Y., X-ray directional dichroism of a polar ferrimagnet. Phys. Rev. Lett. 2004, 92 (13), 137401.

4. Sessoli, R.; Boulon, M.-E.; Caneschi, A.; Mannini, M.; Poggini, L.; Wilhelm, F.; Rogalev, A., Strong magnetochiral dichroism in a paramagnetic molecular helix observed by hard X-rays. Nature Physics 2014.

5. Rinehart, J. D.; Long, J. R., Exploiting single-ion anisotropy in the design of f-element single-molecule magnets. Chemical Science 2011, 2 (11), 2078-2085.

6. Mihalcea, I.; Zill, N.; Mereacre, V.; Anson, C. E.; Powell, A. K., Spontaneous resolution in homochiral helical [Ln (nic) 2 (Hnic) $\left.\left(\mathrm{NO}_{3}\right)\right]$ coordination polymers constructed from a rigid non-chiral organic ligand. Crystal Growth $\mathcal{E}$ Design 2014, 14 (9), 4729-4734.

7. Liu, S.; Li, L.-L.; Li, H.; Gao, H.-L.; Cui, J.-Z.; Cheng, P., Slow magnetic relaxation in a lanthanide helix chain compound [Dy (HNA)(NA) 2 (NO 3)] n (HNA= nicotinic acid). Dalton Transactions 2015, 44 (13), 6169-6174.

8. Cornia, A.; Gatteschi, D.; Sessoli, R., New Experimental Techniques for Magnetic Anisotropy in Molecular Materials. Coord. Chem. Rev. 2001, 219, 573-604. 9. Rogalev, A.; Goulon, J.; Goulon-Ginet, C.; Malgrange, C., Instrumentation developments for polarization dependent $\mathrm{x}$-ray spectroscopies. In Magnetism and Synchrotron Radiation, Springer: 2001; pp 6o-86.

10. (a) Perfetti, M.; Cucinotta, G.; Boulon, M. E.; El Hallak, F.; Gao, S.; Sessoli, R., Angular-Resolved
Magnetometry Beyond Triclinic Crystals Part II: Torque Magnetometry of $\mathrm{Cp}^{*}$ ErCOT Single-Molecule Magnets. Chem. Eur. J. 2014, 20, 14051-14056; (b) Perfetti, M.; Lucaccini, E.; Sorace, L.; Costes, J. P.; Sessoli, R., Determination of Magnetic Anisotropy in the LnTRENSAL Complexes ( $\mathrm{Ln}=\mathrm{Tb}$, Dy, Er) by Torque Magnetometry. Inorg. Chem. 2015, 54 (7), 3090-3092; (c) Lucaccini, E.; Briganti, M.; Perfetti, M.; Vendier, L.; Costes, J. P.; Totti, F.; Sessoli, R.; Sorace, L., Relaxation dynamics and magnetic anisotropy in a low symmetry Dy(III) complex. Chem. Eur. J. 2016.

11. Rigamonti, L.; Cornia, A.; Nava, A.; Perfetti, M.; Boulon, M.-E.; Barra, A.-L.; Zhong, X.; Park, K.; Sessoli, R., Mapping of single-site magnetic anisotropy tensors in weakly coupled spin clusters by torque magnetometry. PCCP 2014.

12. Abragam, A.; Bleaney, B., Electron Paramagnetic Resonance of Transition Ions. Dover: New York, 1986.

13. Chilton, N. F.; Collison, D.; McInnes, E. J.; Winpenny, R. E.; Soncini, A., An electrostatic model for the determination of magnetic anisotropy in dysprosium complexes. Nat. Commun. 2013, 4, 2551.

14. (a) Chen, Q.; Meng, Y.-S.; Zhang, Y.-Q.; Jiang, S.-D.; Sun, H.-L.; Gao, S., A 1D dysprosium chain with slow magnetic relaxation constructed from a pyridine- $\mathrm{N}$-oxide ligand. Chem. Commun. 2014, 50 (72), 10434-10437; (b) Brunet, G.; Habib, F.; Korobkov, I.; Murugesu, M., Slow Magnetic Relaxation Observed in Dysprosium Compounds Containing Unsupported Near-Linear Hydroxo-and FluoroBridges. Inorg. Chem. 2015, 54 (13), 6195-6202.

15. Bogani, L.; Caneschi, A.; Fedi, M.; Gatteschi, D.; Massi, M.; Novak, M. A.; Pini, M. G.; Rettori, A.; Sessoli, R.; Vindigni, A., Finite-Size Effects in Single Chain Magnets: an Experimental and Theoretical Study. Phys. Rev. Lett. 2004, 92 (20), art. no.-207204.

16. Giorgetti, C.; Dartyge, E.; Brouder, C.; Baudelet, F.; Meyer, C.; Pizzini, S.; Fontaine, A.; Galéra, R.-M., Quadrupolar effect in X-ray magnetic circular dichroism. Phys. Rev. Lett. 1995, 75 (17), 3186.

17. Alagna, L.; Prosperi, T.; Turchini, S.; Goulon, J.; Rogalev, A.; Goulon-Ginet, C.; Natoli, C. R.; Peacock, R. D.; Stewart, B., X-ray natural circular dichroism. Phys. Rev. Lett. 1998, 8 o (21), 4799. 
